\title{
Predicting parenchymal hematoma associated with endovascular thrombectomy for acute occlusion of anterior circulation large vessel: the GuEss-MALiGn scale
}

\author{
Juhyeon Kim, MD; Chang Hun Kim, MD; Jongsoo Kang, MD; \\ Oh-Young Kwon, MD, PhD
}

Department of Neurology and Institute of Health Science, Gyeongsang National University Hospital, Gyeongsang National University College of Medicine, Jinju, Republic of Korea \\ Care \\ ORIGINAL ARTICLE \\ Received: October 17, 2019 \\ Revised: December 10, 2019 \\ Accepted: December 10, 2019 \\ Corresponding Author: \\ Oh-Young Kwon, MD, PhD \\ Department of Neurology, Gyeongsang \\ National University Hospital, \\ Gyeongsang National University School of Medicine, 79 Gangnam-ro, Jinju \\ 52727, Republic of Korea \\ Tel: +82-55-750-8288 \\ Fax: +82-55-750-1709 \\ E-mail: oykwon@gnu.ac.kr}

\begin{abstract}
Background: Endovascular thrombectomy (EVT) is an emergency treatment for stroke caused by anterior circulation large vessel occlusion (ACLVO). This study aimed to identify the predictors for post-EVT parenchymal hematoma (PH) and to develop a predictive tool using the identified factors.

Methods: Using the clinical and imaging data of consecutive patients with acute ACLVO who underwent EVT, we performed a multivariate binary logistic regression analysis to identify predictors for $\mathrm{PH}$. With the predictors proved by the regression, we developed a scale for predicting PH using receiver operating characteristic (ROC) curve analyses.

Results: In 233 enrolled patients, the mean age was 72.3 years old, and the male proportion was 46.4\%. The rate of PH after EVT was 18.0\%: the rate of type $1 \mathrm{PH}$ was $12.9 \%$, and the rate of type $2 \mathrm{PH}$ was $5.2 \%$. The significant predictors for PH were basal ganglia involvement, embolism, male sex, antihyperlipidemic use, lobar infarction, and serum glucose level. We developed the GuEss-MALiGn scale with the six significant predictors. Each of these six items was placed on a Likert scale and scored as a 0 or 1 . The ROC curve analysis revealed that the area under the curve was 0.771 . The cutoff score for the risk of PH was $>3$. The sensitivity was $59.5 \%$, and the specificity was $78.0 \%$.

Conclusion: We propose the GuEss-MALiGn scale as a tool for predicting PH associated with EVT. Future external validation is needed to determine the reliability of this scale.
\end{abstract}

Keywords: Cerebral infarction; Middle cerebral artery; Thrombectomy; Endovascular procedures; Postoperative complications; Cerebral hemorrhage

\section{INTRODUCTION}

Endovascular thrombectomy (EVT) is an intraarterial treatment for acute cerebral infarction due to anterior circulation large vessel occlusions (ACLVOs). The reperfusion accomplished by
EVT may prevent the progression of cerebral infarction caused by the occluded vessels. Prior randomized controlled studies have shown that EVT could be the standard care for patients with ACLVO [1-5].

Parenchymal hematoma $(\mathrm{PH})$ may occur in association with

(C) 2020 The Korean Neurocritical Care Society

This is an Open Access article distributed under the terms of the Creative Commons Attribution Non-Commercial License (http://creativecommons.org/licenses/by-nc/4.0/) which permits unrestricted noncommercial use, distribution, and reproduction in any medium, provided the original work is properly cited. 
EVT for ACLVO. The posttreatment PH may negatively impact the outcome of patients who have undergone EVT. A multicenter study of 13 stroke centers retrospectively gathered patients who underwent EVT to treat ACLVO. The incidence of posttreatment $\mathrm{PH}$ increased with an odds ratio of 3.53 in patients with EVT for ACLVO. The occurrence rate of posttreatment $\mathrm{PH}$ was $8.5 \%$. In the multicenter study, atrial fibrillation was a risk factor for $\mathrm{PH}$, but intraarterial tissue plasminogen activator (IA tPA) reduced the occurrence of $\mathrm{PH}$ [6]. Another single-center study also presented a 9.0\% prevalence of posttreatment $\mathrm{PH}$ after EVT for an ACLVO. In that study, hyperlipidemia and successful reperfusion reduced the occurrence of $\mathrm{PH}$, while hypertension and longer procedure duration increased the occurrence of $\mathrm{PH}[7]$.

The European Cooperative Acute Stroke Study (ECASS) categorized hemorrhagic transformation (HTR) into hemorrhagic infarction (HI) and $\mathrm{PH}[8]$. For the patients treated by EVT, the impact of $\mathrm{PH}$ is more crucial than that of $\mathrm{HI}[6]$; therefore, we focused on PH for this study.

A few studies have examined the factors that predict the $\mathrm{PH}$ after treatment with EVT in patients with ACLVO. The primary purpose of this study is to find the predictors of post-treatment $\mathrm{PH}$ associated with EVT. The secondary purpose of this study is to develop a tool that can be used to predict the risk of developing $\mathrm{PH}$ after performing EVT for ACLVO.

\section{METHODS}

\section{Patient selection}

Consecutive patients with acute ACLVO treated by EVT at the Cerebrovascular Center at Gyeongsang National University Hospital were enrolled retrospectively. The recruitment period was from February 2016 to November 2018. We included patients only when the occlusion site was the internal carotid artery (ICA) or the M1 segment of the middle cerebral artery (MCA). Unilateral occlusions of an anterior cerebral artery (ACA) were not enrolled in this study because we do not perform EVT in those cases. Patients treated with EVT in this study were those who were within 24 hours after ACLVO and had sufficient ischemic penumbra as evaluated by brain imaging.

We excluded patients with any of the following: no initial magnetic resonance imaging (MRI), no follow-up MRI within 1 week, and in-hospital stroke. The reason for the exclusion of the patients with an in-hospital stroke was the lack of data on the initial laboratory findings and initial blood pressure. Finally, 233 patients were enrolled in this study.

\section{Extraction of data}

The demographic and clinical information obtained from the electronic medical records are summarized in Table 1 . The variables associated with the EVT procedures were also from the medical records and are summarized in Table 2. The demographic and clinical information were as follows: demographics (age, sex), infarction state (basal ganglia involvement, occlusion location, embolism, initial pattern of infarction, and National Institute Health Stroke Scale [NIHSS] score at admission time), medical history (hypertension, diabetes, prior stroke or transient ischemic attack, smoking, coronary heart disease, atrial fibrillation, and/or hyperlipidemia), and current medication (oral anticoagulants, antiplatelet drugs, antihypertensive drugs, antidiabetic drugs, and/ or statins). In this study, the subtypes of ischemic stroke were classified into two categories, embolic stroke, and large artery disease. Embolic strokes included cardiogenic embolism and embolic stroke of undetermined source [9].

The extracted variables associated with the EVT procedure were as follows: initial laboratory and clinical findings (serum glu-

Table 1. Demographic and baseline clinical characteristics of the enrolled patients $(n=233)$

\begin{tabular}{lccc}
\hline Characteristic & $\begin{array}{c}\text { Non-PH group } \\
(\mathrm{n}=191)\end{array}$ & $\begin{array}{c}\text { PH group } \\
(\mathrm{n}=42)\end{array}$ & Pvalue \\
\hline Age (yr) & $72.4 \pm 11.6$ & $71.8 \pm 9.6$ & 0.774 \\
Male sex & $84(44.0)$ & $24(57.1)$ & 0.128 \\
Location of thrombus & & & 0.855 \\
$\quad$ Internal carotid artery & $57(29.8)$ & $13(31.0)$ & \\
$\quad$ Middle cerebral artery & $134(70.2)$ & $29(69.0)$ & \\
Embolism & $136(71.2)$ & $36(85.7)$ & 0.263 \\
Basal ganglia involvement & $126(66.0)$ & $36(85.7)$ & 0.015 \\
Lobar infarction & $79(41.4)$ & $28(66.7)$ & 0.004 \\
NIHSS score, initial & $13.6 \pm 5.2$ & $15.1 \pm 4.2$ & 0.078 \\
Preexisting medication use & & & \\
$\quad$ Antithrombotics & $70(36.6)$ & $17(40.5)$ & 0.725 \\
Antihypertensives & $106(55.5)$ & $25(59.5)$ & 0.732 \\
$\quad$ Antidiabetics & $33(17.3)$ & $13(31.0)$ & 0.054 \\
Antihyperlipidemics & $47(24.6)$ & $16(38.1)$ & 0.086 \\
Smoking history & $41(21.5)$ & $11(26.2)$ & 0.541 \\
Prior medical history & & & \\
$\quad$ Hypertension & $123(64.4)$ & $26(61.9)$ & 0.859 \\
Diabetes & $48(25.1)$ & $15(35.7)$ & 0.181 \\
Hyperlipidemia & $67(35.1)$ & $15(35.7)$ & 1.000 \\
Atrial fibrillation & $90(47.1)$ & $23(54.8)$ & 0.398 \\
Coronary artery disease & $22(11.5)$ & $4(9.5)$ & 1.000 \\
Stroke & $35(18.3)$ & $9(21.4)$ & 0.665 \\
\hline
\end{tabular}

Values are presented as mean \pm SD or number $(\%)$.

$\mathrm{PH}$, parenchymal hematoma; NIHSS, National Institute Health Stroke Scale. 
cose levels, prothrombin time international normalized ratio [PT INR], hemoglobin A1c, and systolic blood pressure), and interventions (intravenous recombinant tissue plasminogen activator, use of balloon guiding catheter, thrombectomy technique, use of tirofiban during EVT, intervals from symptom onset to puncture time, number of retrievals, and procedure time).

In this study, we classified the $\mathrm{PH}$ according to the ECASS criteria [8]. The ECASS classification grades $\mathrm{PH}$ as $\mathrm{PH}$ type 1 ( $\mathrm{PH} 1)$ and $\mathrm{PH}$ type 2 (PH2). $\mathrm{PH} 1$ is the hematoma that occupies less than $30 \%$ of the infarcted area. $\mathrm{PH} 2$ is the hematoma occupying more than $30 \%$ of the infarcted area and has a significant space-occupying effect. To determine the grade of the $\mathrm{PH}$, each of the two authors ( $\mathrm{CHK}$ and $\mathrm{JK})$ evaluated the cases independently. If the decision was not initially concordant between them, a consensus was achieved through discussion.

The state of reperfusion after EVT was defined based on the Treatment in Cerebral Ischemia Scale (TICI) [10]. In the TICI scale, the degree of perfusion after vascular therapy for acute ischemic stroke has five grades: grade 0 , grade 1 , grade $2 a$, grade $2 b$, and grade 3 . Grade 0 means no perfusion, and grade 3 indicates complete reperfusion of the prior occlusion of the target artery and its distal branches. We defined complete reperfusion as the vascular state of TICI grade 3 for this study.

Table 2. Variables associated with endovascular therapy procedures in the enrolled patients $(n=233)$

\begin{tabular}{lccc}
\hline Characteristic & $\begin{array}{c}\text { Non-PH group } \\
(\mathrm{n}=191)\end{array}$ & $\begin{array}{c}\mathrm{PH} \text { group } \\
(\mathrm{n}=42)\end{array}$ & P value \\
\hline IV thrombolysis & $64(33.5)$ & $16(38.1)$ & 0.593 \\
$\begin{array}{c}\text { Onset to puncture time } \\
\text { (min) }\end{array}$ & $408.9 \pm 372.9$ & $385.7 \pm 239.5$ & 0.045 \\
$\begin{array}{l}\text { Systolic BP, initial (mm } \\
\text { Hg) }\end{array}$ & $144.42 \pm 25.125$ & $145.24 \pm 22.978$ & 0.847 \\
PT INR, baseline & $1.06 \pm 0.14$ & $1.09 \pm 0.17$ & 0.225 \\
$\begin{array}{l}\text { Serum glucose level } \geq 145, \\
\text { initial (mg/dL) }\end{array}$ & $49(25.7)$ & $21(50.0)$ & 0.003 \\
$\begin{array}{l}\text { Balloon guiding catheter } \\
\text { Thrombectomy technique }\end{array}$ & $81(42.4)$ & $20(47.6)$ & 0.619 \\
$\quad$ & $102(53.4)$ & $21(52.4)$ & \\
$\quad$ Stent retriever & $50(26.2)$ & $7(16.7)$ & \\
$\quad$ ADAPT & $26(13.6)$ & $12(28.6)$ & \\
$\quad$ Multimodal & $5(2.6)$ & $1(2.4)$ & \\
$\quad$ Other techniques & $18(9.5)$ & 0 & 0.304 \\
Use of tirofiban & $88(46.1)$ & $17(40.5)$ & 0.509 \\
Complete reperfusion (yes) & $84.1 \pm 35.7$ & $83.6 \pm 44.9$ & 0.937 \\
\hline Procedure time (min) & & &
\end{tabular}

Values are presented as number (\%) or mean \pm SD.

$\mathrm{PH}$, parenchymal hematoma; IV, intravenous; $\mathrm{BP}$, blood pressure; $\mathrm{PT}$ INR, prothrombin time-international normalized ratio; ADAPT, a direct aspiration first pass technique.

\section{EVT procedure}

Three interventionists (DSC, CHK, and JK) performed the procedures in all recruited patients according to their shift schedule. They obtained digital subtraction angiography images by placing a guiding catheter at the cervical segment of the ICA or the common carotid artery. Those images were used to evaluate the occlusion site of the cerebral infarction.

For EVT, they used a stent retriever or a direct aspiration catheter with a femoral artery approach under conscious sedation. In the case of using the stent retriever, they inserted the microcatheter under the guide of a microwire. After locating the microcatheter at the distal part of the clot, they captured the clot with the stent retriever deployed across the clot. They then retrieved the stent device through the guide catheter while aspirating with a 50 $\mathrm{mL}$ syringe. In cases of using a direct aspiration catheter, they located the catheter at the proximal end of the clot. In that state, they performed the aspiration using a $50 \mathrm{~mL}$ syringe through the aspiration catheter.

When successful recanalization was not achieved with one of the two devices, they also used the other device. If they found chronic severe stenosis of the target artery, they additionally administered intraarterial tirofiban up to $2 \mathrm{mg}$ to prevent reocclusion. Just after finishing the entire procedure, another angiography was performed to evaluate the degree of recanalization.

\section{Statistical analysis}

We grouped the patients into two groups to compare the extracted factors between the two groups: patients with $\mathrm{PH}$ and patients without PH. For simple comparisons of variables, we used chisquare tests for categorical variables and Student $t$ tests for continuous variables. We converted the glucose level at EVT to a binary variable: $<145$ and $\geq 145 \mathrm{mg} / \mathrm{dL}$. In addition, we calculated the interrater kappa regarding the decision as to the presence or absence of $\mathrm{PH}$ between the two authors ( $\mathrm{CHK}$ and $\mathrm{JK}$ ) to provide the degree of accuracy of the decision.

For the regression analysis, we performed a univariate regression of the extracted variables first to find factors that had significant effects on the occurrence of $\mathrm{PH}$. For this univariate regression, we set the level of significance as $P<0.2$. Next, we performed a binary multivariate logistic regression for the factors against the occurrence of $\mathrm{PH}$. For this multivariate regression, we set the level of significance as $P<0.05$. For these statistical analyses, we used IBM SPSS Statistics software ver. 25 (IBM Corp., Armonk, NY USA).

Through gathering the significant factors from the binary multivariate regression, we developed a clinical inventory tool to predict $\mathrm{PH}$ associated with EVT. For the validation of the inventory 
tool, we performed receiver operating characteristic (ROC) curve analyses using MedCalc software ver. 8.0 (MedCalc Software BVBA, Ostend, Belgium). The ROC curve analyses determined the most appropriate cutoff score when the Youden's J index was the highest, the area under the curve (AUC) value, and other statistical results from the case. Additional statistical analyses were performed to observe how the AUC value changes as the cutoff score changes. Whenever the cutoff score was changed, we added ROC curve analyses repeatedly after artificially converting the GuEss-MALiGn scale score to a binary classification based on the individual case.

\section{Ethical statement}

This study was approved by the Institutional Review Board of the Gyeongsang National University (2019-10-044-001), and the need for written informed consent was waived because of the retrospective nature of the currrent study.

\section{RESULTS}

\section{Characteristics of the patients}

The mean age of the enrolled 233 patients was 72.3 years old, with a standard deviation (SD) of 11.3. One hundred eight of the patients were male (46.4\%). PH after EVT occurred in 18.0\% $(n=42)$ of the enrolled patients. According to the subtype of $\mathrm{PH}$, the occurrence rate was $12.9 \%(\mathrm{n}=30)$ for $\mathrm{PH} 1$ and $5.2 \%$ $(\mathrm{n}=12)$ for PH2. The interrater agreement rate for the decision of $\mathrm{PH}$ was $99.6 \%$ between the two investigators ( $\mathrm{CHK}$ and JK), and the kappa value of the agreement was $0.875 \pm 0.41(P<0.01)$.

Comparisons of demographic and clinical characteristics between the non-PH and $\mathrm{PH}$ groups

The number of enrolled patients who underwent EVT for ACLVO was 233. We summarized the demographic and baseline clinical characteristics with the comparison between the non- $\mathrm{PH}$ and $\mathrm{PH}$ groups in Table 1, Fig. 1. Basal ganglia involvement and lobar infarction were significantly different between the two groups $(P<0.05)$. The percent of basal ganglia involvement was $66.0 \%$ and $85.7 \%$ for the non-PH and $\mathrm{PH}$ groups, respectively. The rate of lobar infarction was $41.4 \%$ and $66.7 \%$ for the non-PH and $\mathrm{PH}$ groups, respectively.

Differences between the two groups for age, sex, location of thrombus, embolic infarction, the NIHSS score at the admission time, previous medication use, smoking history, and previous medical history in the univariate analysis did not reach the level of significance. The mean age of the non-PH and $\mathrm{PH}$ groups was $72.4 \pm 11.6$ and $71.8 \pm 9.6$ years, respectively. For the non- $\mathrm{PH}$ and
PH groups, the male proportion was $44.0 \%$ and $57.1 \%$, respectively; the frequency of embolic infarction was $71.2 \%$, and $85.7 \%$, respectively; and the rate of antihyperlipidemic use was $24.6 \%$ and $38.1 \%$, respectively. The proportion of patients using antithrombotics as a preexisting treatment was $36.6 \%$ for the non- $\mathrm{PH}$ group and $40.5 \%$ for the $\mathrm{PH}$ group.

For patients included in our study, the occlusion was located in either the ICA or the MCA, for $29.8 \%$ and $70.2 \%$, respectively, in the non-PH group, and $31.0 \%$ and $69.0 \%$, respectively, in the $\mathrm{PH}$ group. There was no case where the ACA was the occlusion site. For cases of solitary and unilateral ACA occlusion, we do not perform EVT treatment. For this reason, this study excluded cases with solitary ACA occlusion.

Comparison offactors associated with the EVT procedure between the non-PH and $\mathrm{PH}$ groups

We summarized the factors associated with the EVT procedure with a comparison between the two study groups in Table 2, Fig. 1. The initial glucose level was significantly different between the two groups. The incidence of the initial glucose level equal to or higher than $145 \mathrm{mg} / \mathrm{dL}$ was $25.7 \%$ and $50.0 \%$ in the non-PH and $\mathrm{PH}$ groups, respectively. IV thrombolysis, onset to puncture time, initial systolic blood pressure, baseline INR, balloon guid-

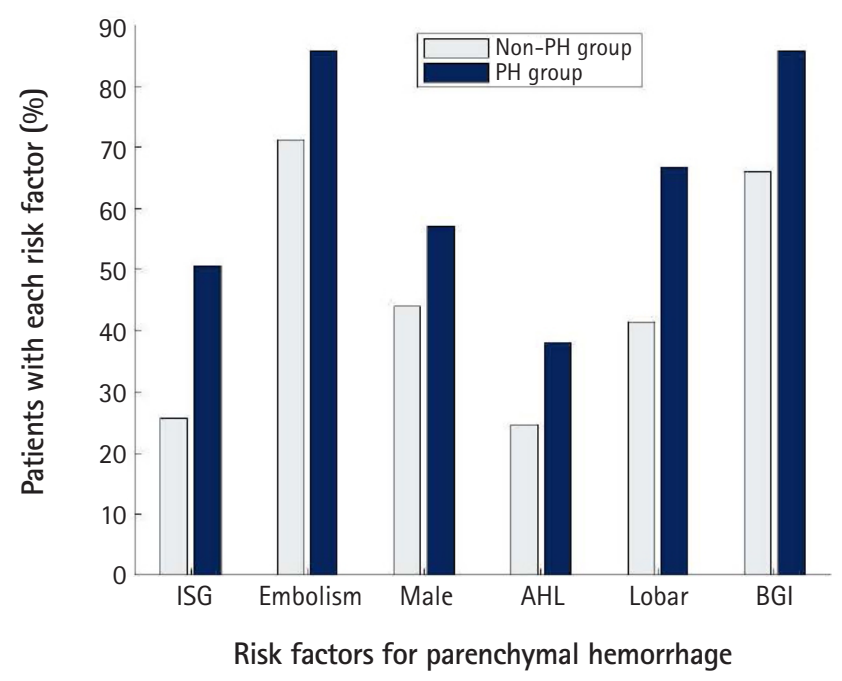

Fig. 1. The occurrence rate of parenchymal hematoma (PH) associated with endovascular therapy (EVT) according to each item of the GuEss-MALiGn scale: comparisons between the non$\mathrm{PH}$ group and the PH group. In patients with acute stroke by the occlusion at the internal carotid artery or the M1 segment of the middle cerebral artery, the six factors were associated with the occurrence of PH associated with EVT. Those factors were: the initial serum glucose (ISG) level, embolism, male sex, antihyperlipidemic (AHL) use, lobar infarction (Lobar), and basal ganglia involvement (BGI). 
ing catheter, thrombectomy technique, use of tirofiban, complete reperfusion, and procedure time were not significantly different between the two groups in the univariate analysis. The onset to puncture time in the non- $\mathrm{PH}$ group was $408.9 \pm 372.9$ minutes, and $385.7 \pm 239.5$ minutes in the $\mathrm{PH}$ group.

\section{Binary logistic regression analysis}

\section{Univariate regression}

Nine factors were significantly associated with the occurrence of PH $(P<0.2)$ : male sex, embolism, basal ganglia involvement, lobar infarction, antidiabetic use, antihyperlipidemic use, diabetes mellitus history, NIHSS score, and initial serum glucose level (Table 3).

\section{Binomial multivariate logistic regression}

Six factors were significant in the multivariate regression combining with the nine factors mentioned above $(P<0.05)$ : initial serum glucose level, embolism, male sex, antihyperlipidemic use, lobar infarction, and basal ganglia involvement.

\section{A clinical inventory tool for predicting $\mathrm{PH}$ associated with EVT}

GuEss-MALiGn scale

We developed a clinical inventory tool to predict $\mathrm{PH}$ associated with EVT, with the six significant factors obtained through multiple regression analysis. We named this as "GuEss-MALiGn scale" using the six upper case characters to indicate the six predictors: serum glucose level at EVT (G), embolic infarction (E), male sex (M), antihyperlipidemic use (A), lobar infarction (L), and basal ganglia involvement $(\mathrm{G})$. All six predictors were binary conversions making it convenient to compose a six-item Likert scale inventory. For each item, the score is 0 or 1 , according to the state of each patient being assessed. Therefore, the range of the total score of the GuEss-MALiGn scale is 0-6.

\section{The optimal cutoff point for the high risk of $\mathrm{PH}$}

For the patients in this study, the mean score of the GuEss-MALiGn scale was $2.9 \pm 1.1$. Table 4 shows the results of the ROC curve analysis of the score of the GuEss-MALiGn scale for predicting a high risk of $\mathrm{PH}$ associated with EVT, and Fig. 2A shows the ROC curve drawn by the analysis. The GuEss-MALiGn scale score was suited for discerning a high risk of $\mathrm{PH}$ associated with

Table 3. Binary logistic regression analysis for independent predictors of parenchymal hematoma associated with endovascular thrombectomy

\begin{tabular}{|c|c|c|c|c|c|c|}
\hline \multirow{2}{*}{ Variable } & \multicolumn{3}{|c|}{ Univariate analysis } & \multicolumn{3}{|c|}{ Multivariate analysis } \\
\hline & OR & $95 \% \mathrm{Cl}$ & $P$ value & OR & $95 \% \mathrm{Cl}$ & $P$ value \\
\hline Male sex & 1.70 & $0.87-3.33$ & 0.12 & 3.16 & $1.39-7.15$ & 0.01 \\
\hline Embolism & 2.44 & $0.98-6.13$ & 0.06 & 3.47 & $1.15-10.48$ & 0.03 \\
\hline Basal ganglia involvement & 3.10 & $1.24-7.73$ & 0.02 & 2.98 & $1.09-8.13$ & 0.03 \\
\hline Lobar infarction & 2.84 & $1.40-5.73$ & 0.00 & 2.94 & $1.30-6.63$ & 0.01 \\
\hline Antidiabetic medications & 2.15 & $1.01-4.56$ & 0.05 & 2.80 & $0.48-16.49$ & 0.26 \\
\hline Antihyperlipidemics & 1.89 & $0.93-3.81$ & 0.08 & 2.56 & $1.13-5.85$ & 0.03 \\
\hline Diabetes history & 1.66 & $0.81-3.37$ & 0.17 & 0.64 & $0.11-3.65$ & 0.62 \\
\hline NIHSS score, initial & 1.07 & $0.99-1.15$ & 0.08 & 1.00 & $0.91-1.09$ & 0.95 \\
\hline Serum glucose, initial & 2.90 & $1.46-5.76$ & 0.00 & 2.56 & $1.09-6.02$ & 0.03 \\
\hline
\end{tabular}

$\mathrm{OR}$, odds ratio; $\mathrm{Cl}$, confidence interval; NIHSS, National Institute Health Stroke Scale.

Table 4. The receiver operating characteristics curve analysis of the GuEss-MALiGn scale for predicting the risk of parenchymal hematoma

\begin{tabular}{|c|c|c|c|c|c|c|c|c|c|}
\hline \multirow{2}{*}{ Cutoff point } & \multicolumn{2}{|c|}{ Sensitivity (\%) } & \multicolumn{2}{|c|}{ Specificity (\%) } & \multirow{2}{*}{ PPV (\%) } & \multirow{2}{*}{ NPV (\%) } & \multicolumn{3}{|c|}{ AUC } \\
\hline & Value & $95 \% \mathrm{Cl}$ & Value & $95 \% \mathrm{Cl}$ & & & Value & $95 \% \mathrm{Cl}$ & $P$ value \\
\hline$>1$ & 100.0 & $91.6-100.0$ & 11.0 & $6.9-16.3$ & 19.8 & 100.0 & & & \\
\hline$>2$ & 95.2 & 83.8-99.4 & 40.8 & $33.8-48.2$ & 26.1 & 97.5 & & & \\
\hline$>3^{a)}$ & 59.5 & $43.3-74.4$ & 78.0 & $71.5-83.7$ & 37.3 & 89.8 & 0.771 & $0.712-0.824$ & $<0.001$ \\
\hline$>4$ & 26.19 & $13.9-42.0$ & 95.81 & $91.9-98.2$ & 57.9 & 85.5 & & & \\
\hline$>5$ & 2.38 & $0.06-12.6$ & 100.0 & $98.1-100.0$ & 100.0 & 82.4 & & & \\
\hline
\end{tabular}

$\mathrm{Cl}$, confidence interval; PPV, positive predictive value; NPV, negative predictive value; $\mathrm{AUC}$, area under the curve.

${ }^{\text {a) }}$ Optimal cutoff point 
(A)

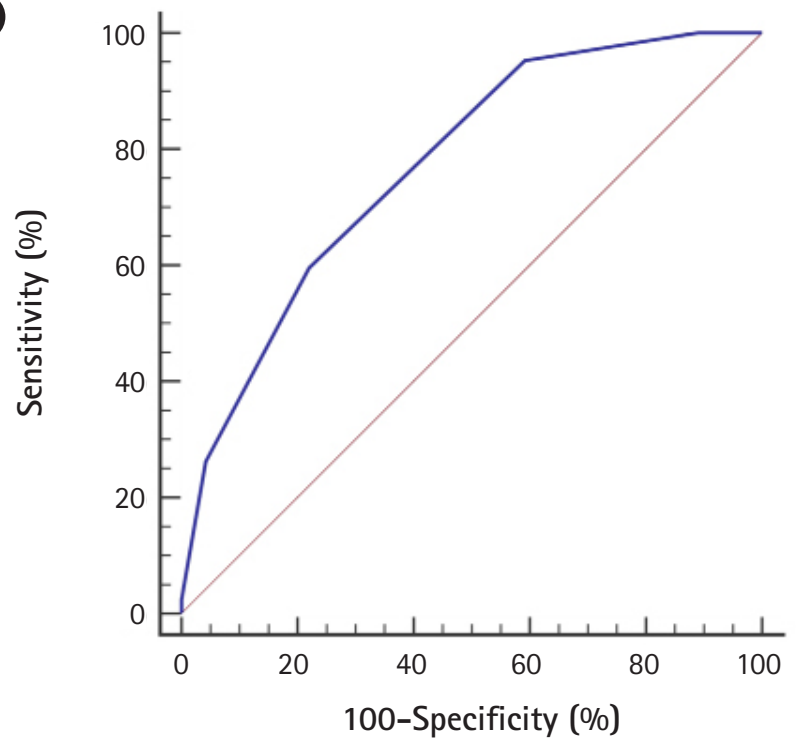

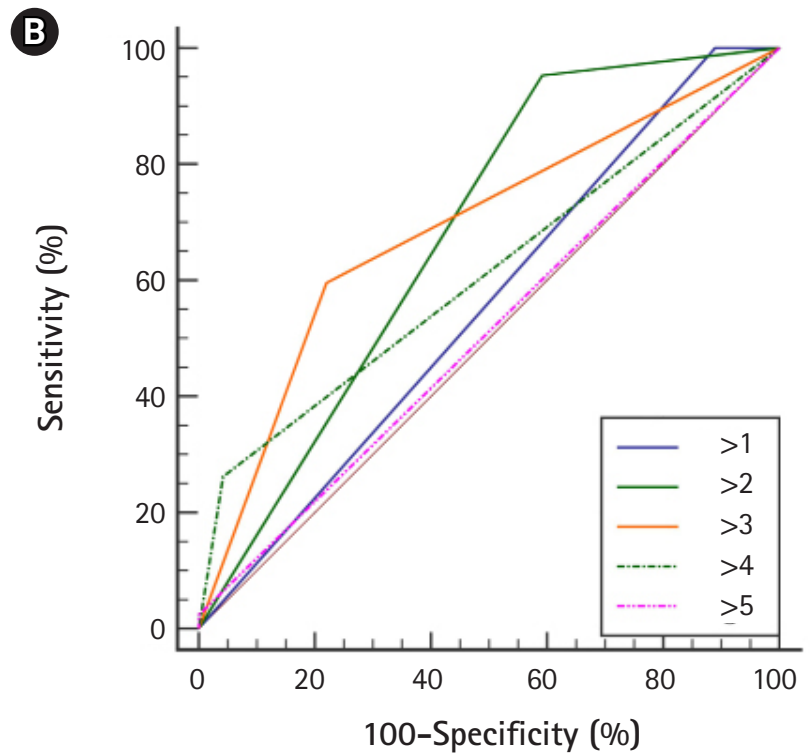

Fig. 2. The receiver operating characteristic (ROC) curves of the score of the GuEss-MALiGn scale for detecting the risk of parenchymal hematoma after EVT of the acute occlusion of anterior circulation large vessel. (A) For detecting the risk, ROC analysis of the score of the GuEss-MALiGn scale determined an area under the curve (AUC) of 0.771. At a cutoff score of $>3$, the sensitivity and specificity for detecting the risk were $59.5 \%$ and $78.0 \%$, respectively. (B) When comparing the AUC values between the different scores in the cases that they were hypothetically the GuEss-MALiGn cutoff points, the AUC value was also the highest at 0.688 when the cutoff point was $>3$.

Table 5. Comparison of AUC values between the various scores when they were hypothetically the GuEss-MALiGn cutoff point

\begin{tabular}{lccc}
\hline Hypothetical cutoff point & AUC & SE & $95 \% \mathrm{Cl}$ \\
\hline$>1$ & 0.555 & 0.0113 & $0.489-0.620$ \\
$>2$ & 0.680 & 0.0244 & $0.616-0.740$ \\
$>3$ & 0.688 & 0.0412 & $0.624-0.747$ \\
$>4$ & 0.610 & 0.0351 & $0.544-0.673$ \\
$>5$ & 0.512 & 0.0119 & $0.446-0.578$ \\
\hline
\end{tabular}

$\mathrm{AUC}$, area under the curve; $\mathrm{SE}$, standard error; $\mathrm{Cl}$, confidence interval.

EVT (AUC, $0.771 ; P<0.0001 ; 95 \%$ confidence interval, 0.712 to 0.824 ) (Table 4, Fig. 2A). The optimal cutoff point was $>3$. At the cutoff point of $>3$, the sensitivity and specificity were $59.5 \%$ and $78.0 \%$, respectively, with a positive predictive value of $37.3 \%$ and a negative predictive value of $85.5 \%$. To observe how the AUC changes as the cutoff score shifts, we conducted additional statistical analyses. Even in this comparison between the different scores, when the cutoff point was $>3$, the AUC value was at its highest, at 0.688 (Table 5, Fig. 2B).

\section{DISCUSSION}

Posttreatment $\mathrm{PH}$ associated with EVT results in adverse impacts on the outcome of patients with ACLVO. PH increased poor functional outcomes by 6.24 times and mortality by 3.53 times in a multicenter study [6]. Through ROC curve analyses, we proved that the GuEss-MALiGn scale was clinically useful to predict the posttreatment $\mathrm{PH}$. The optimal cutoff point of the GuEss-MALiGn scale for predicting the $\mathrm{PH}$ was more than 3 points. The GuEss-MALiGn scale may help us identify patients with a high risk of $\mathrm{PH}$ associated with EVT. We could provide particular attention and effort toward reducing $\mathrm{PH}$ for the patients determined to be at high risk by the GuEss-MALiGn scale.

Studies regarding the occurrence rate of $\mathrm{PH}$ associated with EVT in patients with ACLVO are scant. Nogueira et al. [6] gathered patients with ACLVO who underwent EVT within 8 hours after EVT from 13 stroke centers. The rate of $\mathrm{PH}$ associated with EVT was 8.5\% among the enrolled patients [6]. Lee et al. [7] also recruited patients with EVT for ACLVO in a single-center study, and the rate for $\mathrm{PH}$ was $9.0 \%$ in their collection. In our study, $\mathrm{PH}$ occurred in $18.0 \%$ of enrolled patients. There was a marked difference in the rate between our study and the previous two studies.

There are a number of potential reasons for the difference in the occurrence rate of $\mathrm{PH}$ associated with EVT between the previous studies and our study. One plausible explanation is that the imaging modalities for determining the occurrence of $\mathrm{PH}$ were different among the studies. Small PHs, which are not easy to detect with computed tomography (CT), will be detected more frequently by MRI. Nogueira et al. [6] evaluated the PH associated with EVT with CT as well as MRI, and Lee et al. [7] used only CT. Meanwhile, we defined the occurrence of PH using MRI in 
all enrolled cases. Considering the difference in imaging modalities, we calculated the occurrence rate of $\mathrm{PH} 2$. $\mathrm{PH} 2$, whose size was defined as more than $30 \%$ of the infarcted area, occurred in $5.2 \%$ of enrolled patients in our study. The occurrence rate of $\mathrm{PH} 2$ was less than that of $\mathrm{PH}$ in the two previous studies $[6,7]$. The two previous studies did not report the occurrence rates of $\mathrm{PH} 2$. Visual inspection may similarly detect massive bleeding between the CT-based and MRI-based decision paradigms, so our incidence of $\mathrm{PH} 2$ might have been similar to the previous studies.

In the study by Nogueira et al. [6], the use of IA tPA was a beneficial factor in reducing the posttreatment $\mathrm{PH}$. In contrast, the effect of IA tPA could not be evaluated in our research because we did not use IA tPA in our enrolled patients. The lack of IA tPA use might explain the higher rate of $\mathrm{PH}$ in our study than in their previous study. The occurrence rate of the $\mathrm{PH} 2$ subset in our study was less than that of total $\mathrm{PH}$ in the previous two studies. The use of IA tPA might have reduced the occurrence of small hemorrhages in the previous study because the occurrence rate was markedly less than that in our research.

Six items in the GuEss-MALiGn scale are known to be associated with the HTR of cerebral infarction. A previous study also showed that the basal ganglia infarction occurred before the EVT treatment and was associated with the risk of HTR in patients with ACLVO [11]. HTR is associated with reperfusion after occlusion on the perforators, which are more vulnerable to blood brain barrier failure than larger arteries are [12]. Hyperperfusion after reperfusion and restoration of blood pressure associated with embolic infarction can damage brain parenchyma and cerebral vessel walls [13]. Therefore, embolism is a risk factor for posttreatment $\mathrm{PH}$, and its effect was independent of anticoagulant or antithrombotic use $[14,15]$. Intracerebral hemorrhage in the general population was more prevalent in males than females in an epidemiological study performed in Japan [16]. As shown in the results of our study, the $\mathrm{PH}$ associated EVT treatment may also occur more frequently in males.

Hyperlipidemia is a beneficial factor for the prevention of posttreatment $\mathrm{PH}[7,17]$. A low level of serum cholesterol can enhance the necrosis of smooth muscle cells in the medial layer of the artery. The vascular endothelium, damaged by the low level of serum cholesterol, may be an ideal site for the development of microaneurysms [18]. Brain edema caused by massive cerebral infarction compresses and damages the vasculatures peripheral to the volume of the infarction. Delayed perfusion that occurs after resolution of the edema increases the likelihood of HTR in damaged blood vessels [19-22]. Hyperglycemia causes diffuse damage in the microvasculature, increasing the volume of the cerebral infarction. The increment of infarct volume increases the risk of
HTR [23-26].

There were several limitations to this study. Firstly, the most significant limitation of our proposed GuEss-MALiGn scale is that there has not yet been external validation. In the future, additional validation performed with patients extracted from other populations will improve the efficacy of the GuEss-MALiGn scale. Secondly, the sensitivity of the GuEss-MALiGn scale was relatively low at $59.5 \%$ for detecting PH after EVT. Contrastingly, the specificity was a reasonable $78.0 \%$, and this may make the GuEss-MALiGn scale clinically significant when the score indicates a high risk of PH occurrence. Thirdly, we did not obtain the Alberta Stroke Program Early CT Score (ASPECTS), which is a surrogate for infarction volume. Nevertheless, in this study, we analyzed the involvement of the basal ganglia and lobar infarction as substitutes for the ASPECTS score. Both were significant EVT-related risk factors for PH. Fourthly, there may have been bias in the patient selection as this study was a retrospective study from a single center. Therefore, there is a possibility that cultural aspects deriving from the local area might have affected the outcome results. Lastly, a difference in the skills among the interventionists might have affected the outcome of EVT.

\section{ARTICLE INFORMATION}

\section{Conflict of interest}

Dr. OY Kwon is an editorial board member of the journal but was not involved in the peer reviewer selection, evaluation, or decision process of this article. There are no other potential conflicts of interest relevant to this article to declare.

\section{ORCID}

Juhyeon Kim, https://orcid.org/0000-0001-6466-375X

Chang Hun Kim, https://orcid.org/0000-0003-1895-1595

Jongsoo Kang, https://orcid.org/0000-0003-4359-6214

Oh-Young Kwon, https://orcid.org/0000-0001-9576-1926

\section{Author contributions}

Conceptualization: CHK, JK, and OYK. Data curation \& Formal analysis: JHK, CHK, JK, and OYK. Visualization \& Writingoriginal draft: JHK, CHK, JK, and OYK. Writing-review editing: JHK, CHK, JK, and OYK.

\section{REFERENCES}

1. Berkhemer OA, Fransen PS, Beumer D, van den Berg LA, Lingsma HF, Yoo AJ, et al. A randomized trial of intraarterial treatment for acute ischemic stroke. N Engl J Med 2015;372:11-20. 
2. Goyal M, Demchuk AM, Menon BK, Eesa M, Rempel JL, Thornton J, et al. Randomized assessment of rapid endovascular treatment of ischemic stroke. N Engl J Med 2015;372:1019-30.

3. Jovin TG, Chamorro A, Cobo E, de Miquel MA, Molina CA, Rovira A, et al. Thrombectomy within 8 hours after symptom onset in ischemic stroke. N Engl J Med 2015;372:2296-306.

4. Saver JL, Goyal M, Bonafe A, Diener HC, Levy EI, Pereira VM, et al. Stent-retriever thrombectomy after intravenous t-PA vs. t-PA alone in stroke. N Engl J Med 2015;372:2285-95.

5. Campbell BC, Mitchell PJ, Kleinig TJ, Dewey HM, Churilov L, Yassi N, et al. Endovascular therapy for ischemic stroke with perfusion-imaging selection. N Engl J Med 2015;372:1009-18.

6. Nogueira RG, Gupta R, Jovin TG, Levy EI, Liebeskind DS, Zaidat OO, et al. Predictors and clinical relevance of hemorrhagic transformation after endovascular therapy for anterior circulation large vessel occlusion strokes: a multicenter retrospective analysis of 1122 patients. J Neurointerv Surg 2015; 7:16-21.

7. Lee YB, Yoon W, Lee YY, Kim SK, Baek BH, Kim JT, et al. Predictors and impact of hemorrhagic transformations after endovascular thrombectomy in patients with acute large vessel occlusions. J Neurointerv Surg 2019;11:469-73.

8. Fiorelli M, Bastianello S, von Kummer R, del Zoppo GJ, Larrue V, Lesaffre E, et al. Hemorrhagic transformation within 36 hours of a cerebral infarct: relationships with early clinical deterioration and 3-month outcome in the European Cooperative Acute Stroke Study I (ECASS I) cohort. Stroke 1999;30:2280-4.

9. Hart RG, Catanese L, Perera KS, Ntaios G, Connolly SJ. Embolic stroke of undetermined source: a systematic review and clinical update. Stroke 2017;48:867-72.

10. Higashida RT, Furlan AJ, Roberts H, Tomsick T, Connors B, Barr J, et al. Trial design and reporting standards for intra-arterial cerebral thrombolysis for acute ischemic stroke. Stroke 2003; 34:e109-37.

11. Loh Y, Towfighi A, Liebeskind DS, MacArthur DL, Vespa P, Gonzalez NR, et al. Basal ganglionic infarction before mechanical thrombectomy predicts poor outcome. Stroke 2009;40: 3315-20.

12. Bang OY, Saver JL, Alger JR, Shah SH, Buck BH, Starkman S, et al. Patterns and predictors of blood-brain barrier permeability derangements in acute ischemic stroke. Stroke 2009;40:454-61.

13. del Zoppo GJ, von Kummer R, Hamann GF. Ischaemic damage of brain microvessels: inherent risks for thrombolytic treatment in stroke. J Neurol Neurosurg Psychiatry 1998;65:1-9.

14. Wahlgren N, Ahmed N, Eriksson N, Aichner F, Bluhmki E, Dávalos A, et al. Multivariable analysis of outcome predictors and adjustment of main outcome results to baseline data profile in randomized controlled trials: Safe Implementation of Thrombolysis in Stroke-MOnitoring STudy (SITS-MOST). Stroke 2008;39:3316-22.

15. Okada Y, Yamaguchi T, Minematsu K, Miyashita T, Sawada T, Sadoshima S, et al. Hemorrhagic transformation in cerebral embolism. Stroke 1989;20:598-603.

16. Nagura J, Suzuki K, Hayashi M, Sakamoto T, Shindo K, Oishi H, et al. Stroke subtypes and lesion sites in Akita, Japan. J Stroke Cerebrovasc Dis 2005; 14:1-7.

17. Wang X, Dong Y, Qi X, Huang C, Hou L. Cholesterol levels and risk of hemorrhagic stroke: a systematic review and meta-analysis. Stroke 2013;44:1833-9.

18. Konishi M, Iso H, Komachi Y, Iida M, Shimamoto T, Jacobs DR Jr, et al. Associations of serum total cholesterol, different types of stroke, and stenosis distribution of cerebral arteries: the Akita Pathology Study. Stroke 1993;24:954-64.

19. Kim JH, Bang OY, Liebeskind DS, Ovbiagele B, Kim GM, Chung CS, et al. Impact of baseline tissue status (diffusion-weighted imaging lesion) versus perfusion status (severity of hypoperfusion) on hemorrhagic transformation. Stroke 2010; 41:e135-42.

20. Tong DC, Adami A, Moseley ME, Marks MP. Prediction of hemorrhagic transformation following acute stroke: role of diffusion- and perfusion-weighted magnetic resonance imaging. Arch Neurol 2001;58:587-93.

21. Wang BG, Yang N, Lin M, Lu B. Analysis of risk factors of hemorrhagic transformation after acute ischemic stroke: cerebral microbleeds do not correlate with hemorrhagic transformation. Cell Biochem Biophys 2014;70:135-42.

22. Kerenyi L, Kardos L, Szász J, Szatmári S, Bereczki D, Hegedüs K, et al. Factors influencing hemorrhagic transformation in ischemic stroke: a clinicopathological comparison. Eur J Neurol 2006; 13:1251-5.

23. Hafer-Macko CE, Ivey FM, Gyure KA, Sorkin JD, Macko RF. Thrombomodulin deficiency in human diabetic nerve microvasculature. Diabetes 2002;51:1957-63.

24. Ceriello A, Giugliano D, Quatraro A, Marchi E, Barbanti M, Lefèbvre P. Evidence for a hyperglycaemia-dependent decrease of antithrombin III-thrombin complex formation in humans. Diabetologia 1990;33:163-7.

25. Ceriello A, Giacomello R, Stel G, Motz E, Taboga C, Tonutti L, et al. Hyperglycemia-induced thrombin formation in diabetes: the possible role of oxidative stress. Diabetes 1995;44:924-8.

26. Pandolfi A, Cetrullo D, Polishuck R, Alberta MM, Calafiore A, Pellegrini $G$, et al. Plasminogen activator inhibitor type 1 is increased in the arterial wall of type II diabetic subjects. Arterioscler Thromb Vasc Biol 2001;21:1378-82. 